\title{
MMW-NOTFALLCHECKLISTE
}

\section{Traumatische Fingeramputation}

\author{
M. Millrose, M. GeSSLEin, A. EISEnSChenK
}

\author{
Ein 32 Jahre alter Mann schneidet sich beim Teppichverlegen in \\ den Daumen der linken Hand, der dabei auf Höhe des Ballens \\ abgetrennt wird. Das Rettungsdienstpersonal bringt den Finger \\ zusammen mit dem Patienten in die Klinik.
}

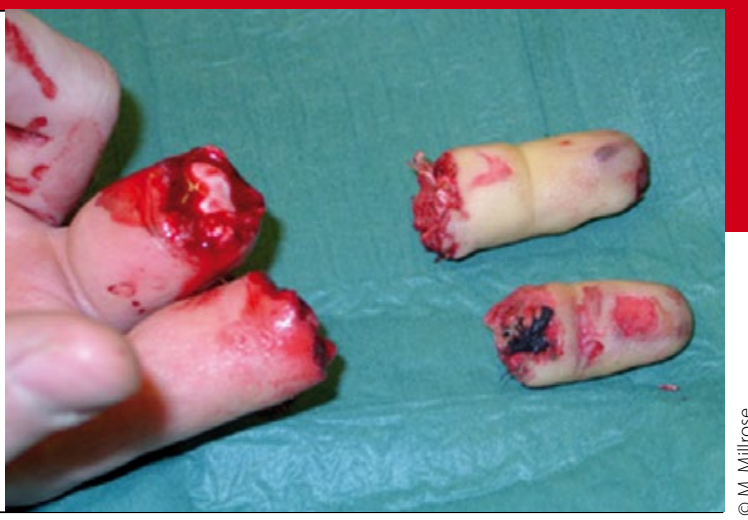

- Der unfallbedingte Verlust eines Fingers oder von Teilen der Hand stellt nicht nur eine schwere Verletzung, sondern auch ein psychisch belastendes Ereignis dar. Nicht jede Amputation lässt sich durch eine Replantation wiederherstellen.

\section{Einteilung der Amputation}

- Guillotineartige Verletzungen: Amputation mit glatten, scharfkantigen Instrumenten, z. B. Cuttermesser oder Axt. Die Amputationskanten weisen ein geringes Gewebetrauma auf; i. d. R. besteht eine günstige Prognose für eine erfolgreiche Replantation mit guter Funktion.

- Kreissägenverletzungen (ca. 70\% aller Amputationen) weisen trotz der höheren Traumatisierung des Gewebes i. d. R. eine gute Replantierbarkeit auf.

- Avulsionsverletzungen (häufig durch Fingerringe) haben aufgrund der Aushülsung des Gewebes eine sehr hohe Gewebetraumatisierung mit langstreckiger Schädigung von Sehnen, Nerven und Gefäßen. Die neurovaskulären Strukturen sind häufig auf unterschiedlichen Höhen verletzt. Die Replantierbarkeit ist schlecht bis unmöglich. Eine Ausnahme stellt hier der Daumen dar, der i.d. R. erfolgreich replantiert werden kann.

- Explosionsverletzungen weisen die stärkste Gewebetraumatisierung mit Defekten und Verletzung sämtlicher Strukturen auf unterschiedlichen Höhen auf. Nur sehr eingeschränkte Replantierbarkeit, da eine gute Funktion mit Resensibilisierung kaum zu erwarten ist.

\section{Voraussetzungen und Indikationen für eine Replantation}

- Entscheidend ist der Zustand des Amputates sowie des Patienten. Insbesondere bei schwer polytraumatisierten $\mathrm{Pa}$ tienten muss aufgrund instabiler Kreislaufverhältnisse von einer Replantation abgesehen werden, um das Leben durch eine verlängerte OP-Dauer nicht zu gefährden. Es gilt der Grundsatz: „life before limb“.

- Die Anoxämiezeit des Amputates ist ebenfalls ein wichtiger Faktor. Man unterschiedet zwischen

- warmer Ischämiezeit: Zeit ohne Blutversorgung bei Körpertemperatur des Amputates.

- kalter Ischämiezeit: Zeit ohne Blutversorgung bei gekühltem Amputat. Die maximale Ischämiezeit bei Amputaten ohne Muskulatur (z. B. Finger) beträgt ungekühlt acht bis zwölf Stunden und gekühlt bis zu 24 Stunden. Amputate mit Muskulatur (Unterarm o. Ä.) haben deutlich kürze Anoxämiezeiten: max. drei bis sechs Stunden bei guter Kühlung (je nach Amputationshöhe).

- Die korrekte Bergung und Lagerung des Amputats ist daher entscheidend für den Erfolg der Replantation: Amputat in keimfreies Material einwickeln, in einen Plastikbeutel verpacken und diesen in einem zweiten Plastikbeutel mit Wasser und Eiswürfeln transportieren.

- Sinnvolle Indikationen sind:

- Daumenamputation

- Mehrfingeramputation (Zeige- und/ oder Mittelfinger)
- Fingeramputationen distal des Ansatzes der oberflächlichen Beugesehne - Amputationen bei Kindern.

\section{Technik der Replantation}

Für eine erfolgreiche Replantation müssen neben den o.g. Voraussetzungen die nötige Expertise und Technik für mikrochirurgische Operationsverfahren vorhanden sein.

Intraoperativ erfolgt zunächst ein ausgiebiges Débridement mit Identifikation der neurovaskulären Strukturen. Zur Fixation der knöchernen Strukturen werden häufig K-Draht-Osteosynthesen verwendet und ggf. mit einer Cerclage kombiniert. Als nächstes werden die Beugeund Strecksehnen versorgt. Im Anschluss erfolgt die mikrochirurgische Rekonstruktion der Gefäßnervenbündel. Bei Defektverletzungen müssen ggf. Veneninterponate interponiert werden, um eine spannungsfreie Wiederherstellung zu ermöglichen. Eine notwendige Rekonstruktion von Nerven kann ein- oder zweizeitig (ggf. mit N. suralis-Interponat) erfolgen.

Nach spannungsfreiem Hautverschluss wird der replantierte Finger druckfrei und warm gelagert. Das Replantat muss regelmäßig kontrolliert werden, um Komplikationen früh zu erkennen.

\section{Für die Verfasser:}

Dr. med. Michael Millrose, Klinik für Hand-, Replantations- und Mikrochirurgie, Unfallkrankenhaus Berlin, Warener Straße 7, D-12683 Berlin, E-Mail: michael.millrose@ukb.de Koautoren: Dr. Markus Gesslein, Nürnberg, Univ.-Prof. Dr. Andreas Eisenschenk, Greifswald 